University of South Carolina

Scholar Commons

$11-1987$

\title{
Surface-to-Surface Transition via Electromagnetic Coupling of Coplanar Waveguides
}

Robert W. Jackson

David W. Matolak

University of South Carolina - Columbia, matolak@cec.sc.edu

Follow this and additional works at: https://scholarcommons.sc.edu/elct_facpub

Part of the Signal Processing Commons, Systems and Communications Commons, and the VLSI and Circuits, Embedded and Hardware Systems Commons

\section{Publication Info}

Postprint version. Published in IEEE Transactions on Microwave Theory and Techniques, Volume 35, Issue 11, 1987, pages 1027-1032.

(C) IEEE Transactions on Microwave Theory and Techniques, 1987, IEEE Jackson, R., Matolak, D. (1987). Surface-to-Surface Transition via Electromagnetic Coupling of Coplanar Waveguides. IEEE Transactions on Microwave Theory and Techniques, 35(11), 1027-1032. http://dx.doi.org/10.1109/TMTT.1987.1133802

This Article is brought to you by the Electrical Engineering, Department of at Scholar Commons. It has been accepted for inclusion in Faculty Publications by an authorized administrator of Scholar Commons. For more information, please contact digres@mailbox.sc.edu. 


\title{
Surface-to-Surface Transition via Electromagnetic Coupling of Coplanar Waveguides
}

\author{
ROBERT W. JACKSON, MEMBER, IEEE, AND DAVID W. MATOLAK, MEMBER, IEEE
}

\begin{abstract}
A transition is investigated which couples coplanar waveguide on one substrate surface (a motherboard) to coplanar waveguide on another substrate surface (a semiconductor chip or subarray) placed above the first. No wire bonds are necessary. A full-wave analysis using coupled line theory is presented and verified experimentally. The use of this transition for coupling to millimeter-wave integrated circuits is discussed.
\end{abstract}

\section{INTRODUCTION}

$\mathrm{C}$ UURENTLY, MANY microwave and millimeterwave modules (receiver front ends, for example) are made up of submodules which are hybrid or MMIC components such as low-noise amplifiers or phase shifters. Wire bonds are usually used to connect these submodules to each other or to transmission lines which run between modules. It is desirable to minimize this type of connection since it is a labor-intensive process and can degrade circuit performance at very high frequencies. In the future it is likely that many of these wire bonds will be eliminated by combining submodules into larger monolithic units. However, phased antenna arrays using tens of thousands of elements would still have need of transitions between antennas, feeds, or active modules, and it would be convenient to eliminate critical wire bonds as much as possible [1].

In this paper, we investigate a method of electromagnetically coupling from a coplanar waveguide (CPW) on the surface of one substrate to a CPW on the surface of another. Fig. 1 shows the basic structures which are under consideration. In both cases the chip (or subarray) is fed electromagnetically by the transmission line on the motherboard underneath. In Fig. 1(a), both the motherboard and the "chip" are assumed to have a high permittivity $\left(\epsilon_{r}=10\right.$ or 13$)$ with the feed line located on the underside of the motherboard. Alternatively, Fig. 1(b) shows the feed line on the top of a low-permittivity motherboard coupling to a high-permittivity chip. It is more convenient to construct a $50-\Omega \mathrm{CPW}$ feed line in the

Manuscript received March 26, 1987; revised July 3, 1987. This work was supported by the Electronics Laboratory, General Electric Co., Syracuse, NY.

R. W. Jackson is with the Department of Electrical and Computer Engineering, University of Massachusetts, Amherst, MA 01003.

D. W. Matolak is with AT\&T Bell Laboratories, North Andover, MA 01845 .

IEEE Log Number 8716591. first structure. The second structure, however, has a tighter coupling for a given chip thickness and therefore requires less surface area. If the motherboard permittivity in this second structure is much less than the chip permittivity, its effects can be neglected and the analysis of both configurations proceeds in the same manner.

CPW is a good candidate for motherboard transmission lines since it has its principal fields on the substrate surface and thus is easier to couple to structures placed above it. It can also have low losses if its dimensions are chosen properly [2]. On the active surface, CPW has advantages due to its ease of construction and low parasitics [3].

In what follows, the transition is modeled as a set of coupled lines which are analyzed using full-wave techniques. The analysis is used to design transitions which were constructed and measured at $C$ - and $X$-band. Finally, potential designs for millimeter-wave applications are discussed.

\section{ANALYSIS}

\section{A. Coupled Line Analysis}

The structures in Fig. 1 each consist of a four-port coupled line section with one port connected to an input line on one surface, one port connected to an output line on the other surface, and the remaining ports terminated in open circuits. In Fig. 2, three cross sections of the coupling region are shown with current configurations for the three modes which have left-right symmetry. The first mode, called the even coupled coplanar waveguide mode, and the second mode, called the odd mode, are used to analyze the coupler. The third mode, a paraliel-plate mode, has been observed to have no effect within the coupler's bandwidth. By determining the impedance and guide wavelength of each of the first two modes, the impedance parameters of the two-port transition are given by [4]

$$
\begin{aligned}
& z_{11}=-j \frac{\left(Z_{0 e}+Z_{0 o}\right)}{2} \operatorname{ctn} \beta l \\
& z_{12}=-j \frac{\left(Z_{0 e}-Z_{0 o}\right)}{2} \csc \beta l \\
& \beta^{2} \equiv\left(\omega / c_{0}\right)^{2}\left(\epsilon_{\text {eff }, e}+\epsilon_{\text {eff, } o}\right) / 2
\end{aligned}
$$




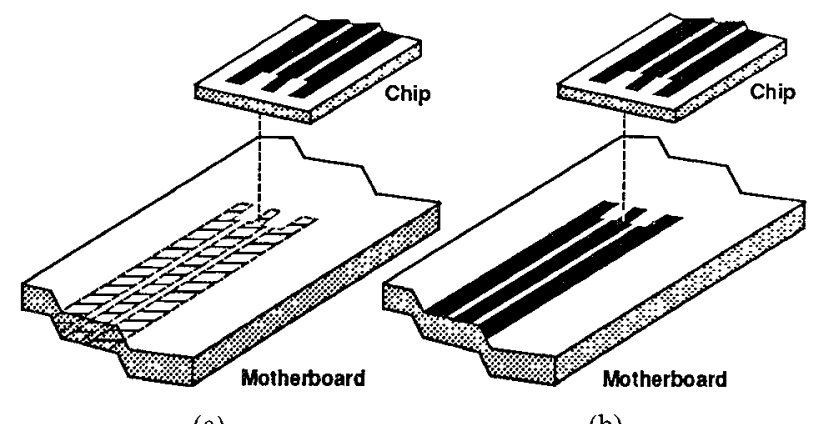

(a)

(b)

Fig. 1. Schematic drawing of motherboard and electromagnetically coupled submodule. (a) Feed line underneath motherboard, both substrates with the same permittivity. (b) Feed line on top of a low-permittivity motherboard.

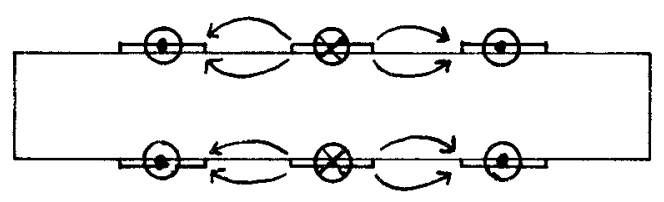

(a)

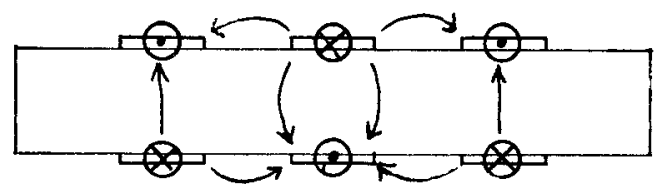

(b)

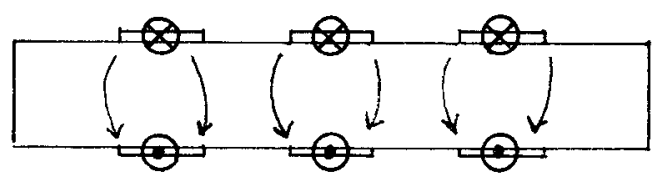

(c)

Fig. 2. Schematic of the three coupled coplanar waveguide modes with left-right symmetry: (a) even mode, (b) odd mode, (c) parallel-plate mode.

where $Z_{0 e}$ and $Z_{0 o}$ are the even- and odd-mode impedances, $\epsilon_{\mathrm{eff}, e}$ and $\epsilon_{\mathrm{eff}, o}$ are the corresponding effective dielectric constants, and $l$ is the coupling length. If $l$ is chosen such that $\beta l$ equals $90^{\circ}$ at the center frequency of the band and the terminating impedance is chosen to be $\left(Z_{0 e}-Z_{0 o}\right) / 2$, a perfect match will occur.

\section{B. Full-Wave Analysis}

In order to determine the odd and even impedances and effective dielectric constants, a full-wave analysis [5], [6] is necessary since the strip widths and separation can become an appreciable fraction of a wavelength in size. The evenmode analysis proceeds by placing a horizontal magnetic wall midway between the two sets of conductors in Fig. 2(a). Likewise, the odd mode is analyzed by placing a horizontal electric wall midway between the two sets of conductors of Fig. 2(b). (The parallel-plate mode in Fig.

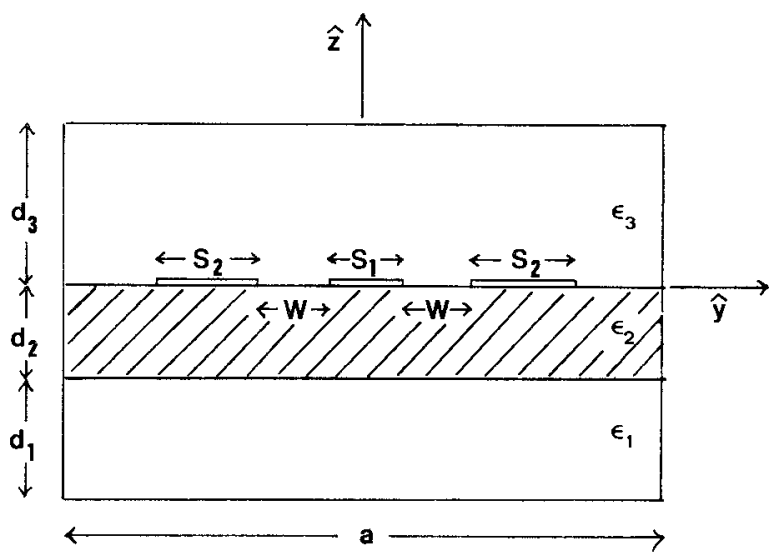

Fig 3. Configuration used in the analysis of the coupled coplanar waveguide. The top and bottom walls are electric or magnetic and the side walls are electric.

2(c) is also obtained this way.) The resulting basic structure is shown in Fig. 3, where an extra dielectric layer has been added so that the effect of air gaps can be estimated. The electric walls on the box sides are set far enough from the CPW to have a negligible effect.

Since the full-wave analysis of structures similar to this is well known [5], [6], it will only be described briefly. As shown in Fig. 3, CPW is enclosed in a box having perfect electric side walls and perfect magnetic or electric walls on the top and bottom. All currents lie on the $z=0$ plane and vary according to the factor $\exp (-j \beta x)$, which is suppressed. The longitudinal current components are assumed to be symmetric around $y=0$ and the transverse components to be antisymmetric. The electric fields tangential to the $z=0$ surface are related to the exciting currents via the expression

$$
\left[\begin{array}{c}
\tilde{E}_{x}(n) \\
\tilde{E}_{y}(n)
\end{array}\right]=\left[\begin{array}{ll}
Q_{x x}(n, \beta) & Q_{x y}(n, \beta) \\
Q_{y x}(n, \beta) & Q_{y y}(n, \beta)
\end{array}\right]\left[\begin{array}{c}
\tilde{J}_{x}(n) \\
\tilde{J_{y}}(n)
\end{array}\right]
$$

where

$$
\begin{aligned}
& \tilde{J_{l}}(n)=\frac{1}{a} \int_{-a / 2}^{a / 2} J_{i}(y) e^{-j n \pi y / a} d y \\
& J_{l}(y)=\sum_{n=-\infty}^{\infty} \tilde{J}_{l}(n) e^{j n \pi y / a}, \quad i=x, y
\end{aligned}
$$

and similar expressions for $\tilde{E}_{l}(n)$ and $E_{l}(y)$. In order that the fields have the proper behavior at the side walls and due to the symmetries in the assumed currents, $n$ takes on only odd values. The derivation of the expressions for $Q_{\imath}(n, \beta)$ is outlined in the Appendix for magnetic and electric cover plates.

Following the usual method of moments approach, $J_{x}$ and $J_{y}$ are expanded in terms of known functions multiplied by unknown coefficients such that

$$
J_{l}(y)=\sum_{l=1}^{N_{t}} A_{t l} f_{l l}(y), \quad i=x \text { or } y
$$

and $N_{i}$ is the number of expansion modes used for each current component. Moments of $E_{x}$ and $E_{y}$ are taken and 
forced to zero on the $z=0$ plane wherever conductors occur such that

$$
\begin{aligned}
\frac{1}{a} \int_{-a / 2}^{a / 2} E_{l}(y) f_{i l}(y) d y & =0, \\
l & =1,2, \cdots, N_{l} \text { and } i=x \text { or } y .
\end{aligned}
$$

This results in the following equations:

$$
\left[\begin{array}{ccc} 
& \vdots & \\
Z^{x x}(\beta) & \vdots & Z^{x y}(\beta) \\
\cdots & \vdots & \cdots \\
Z^{y x}(\beta) & \vdots & Z^{y y}(\beta) \\
& \vdots &
\end{array}\right] \cdot\left[\begin{array}{c}
A_{x, 1} \\
\vdots \\
A_{x}, N_{x} \\
A_{y}, 1 \\
\vdots \\
A_{y}, N_{y}
\end{array}\right]=\left[\begin{array}{c}
\vdots \\
\vdots \\
0 \\
\vdots
\end{array}\right]
$$

where each of the submatrices $Z^{i j}$ is defined by

$$
\begin{gathered}
Z_{l p}^{t j}=\sum_{n=-\infty}^{\infty} \tilde{f_{l l}}(-n) \cdot Q_{\imath j}(n, \beta) \cdot \tilde{f_{j p}}(n), \\
i=x \text { or } y ; j=x \text { or } y ; \\
l=1,2, \cdots, N_{i} ; p=1,2, \cdots, N_{i}
\end{gathered}
$$

and $\tilde{f_{l l}}(n)$ is related to $f_{l l}(y)$ via $(3 \mathrm{a})$. The $i, j$ indices denote the submatrix and the $l, p$ indices denote the element within the submatrix. As usual, $\beta$ is varied until the determinant of the impedance matrix of (6) is zero. The coefficients $A_{l l}$ can then be determined. The forms of the function used in the expansions are [5], [6]

$$
\begin{gathered}
f_{x l}(y)=\frac{\cos m \pi\left(y^{\prime} / S_{k}+.5\right)}{\sqrt{\left(S_{k} / 2\right)^{2}-\left(y^{\prime}\right)^{2}}} \\
f_{y l}(y)=\frac{\sin m \pi\left(y^{\prime} / S_{k}+.5\right)}{\sqrt{\left(S_{k} / 2\right)^{2}-\left(y^{\prime}\right)^{2}}} \\
y^{\prime}=y-y_{k}
\end{gathered}
$$

where $S_{k}$ is the width and $y_{k}$ is the center of the $k$ th strip. So, for example, the $l=1,2$ expansion functions for $J_{x}$ might correspond to $y_{1}=0, k=1$, and $m=0,2$. The $l=$ 3, 4,5 expansion functions for $J_{x}$ would then correspond to $y_{2}= \pm\left(S_{1} / 2+W+S_{2} / 2\right), k=2$, and $m=0,1,2$. Continuing the example, for the $J_{y}$ expansion there would be only one center strip expansion mode identified by $f_{y 1}$ with $y_{1}=0, k=1$, and $m=2$. The side strips would have two modes, $f_{y 2}$ and $f_{y 3}$, with $y_{2}= \pm\left(S_{1} / 2+W+S_{2} / 2\right), k=2$, and $m=1,2$. The symmetries with respect to the $z, x$ plane are used to combine the expansion functions centered at $y_{2}=\left(S_{1} / 2+W+S_{2} / 2\right)$ with the ones centered at $y_{2}=$ $-\left(S_{1} / 2+W+S_{2} / 2\right)$.

Once the propagation constant $\beta$ and the current amplitudes $A_{x l}$ and $A_{y l}$ are determined, the impedance of the resulting mode can be determined according to the power-current definition:

$$
Z_{c}=\frac{1}{\left|I_{0}\right|^{2}}\left[\int_{\mathrm{box}} \int\left(E_{y} H_{z}^{*}-E_{z} H_{y}^{*}\right) d y d z\right]
$$

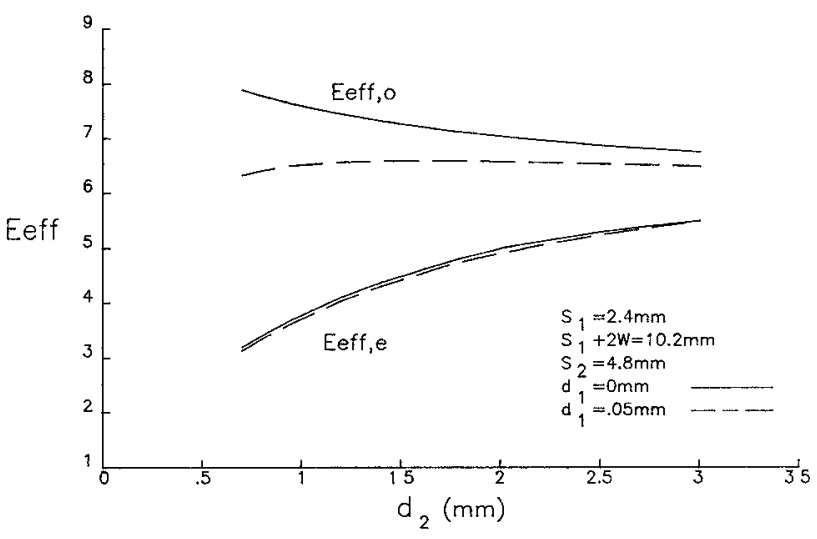

(a)

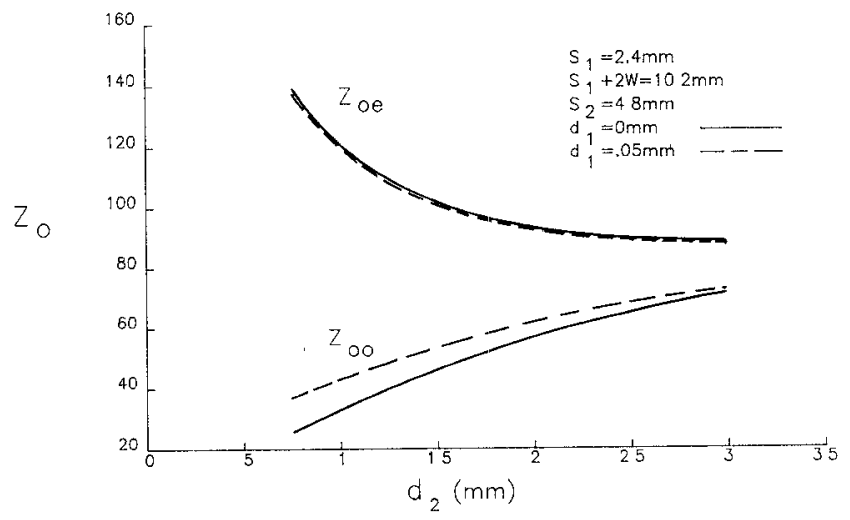

(b)

Fig. 4. Typical computed (a) impedance and (b) effective permittivity without (- $(-)$ and with (---) air gaps.

where $I_{0}$ is the total longitudinal current on the center strip in Fig. 3 and the integration is over the cross section of the box.

The $y$ integration can be converted to a summation in $n$, and the $z$ integration performed analytically. The calculations are straightforward and well known [5] but tedious and, due to space limitations, are not included in this paper.

\section{Representative Results}

To analyze the coupler in Fig. 1(b), the impedance and effective dielectric $\left(\epsilon_{\text {eff }}=\beta^{2} / k_{0}^{2}\right)$ constant of the even (Fig. 2(a)) and odd (Fig. 2(b)) modes must be determined. These quantities can be obtained from the analysis of the structure in Fig. 3 by setting $d_{1}=0, d_{2}=d / 2$, and $d_{3}$ large. For the odd mode, the upper and lower walls are electric; for the even mode, they are magnetic. (The upper wall is of no consequence since it is far away.) A representative result is shown in Fig. 4, where impedances and effective dielectric constant are plotted versus substrate half-thickness $\left(\epsilon_{r}=10.2\right)$. Note that as the substrate thickness increases, the even- and odd-mode impedances move toward each other, indicating, as expected, lower coupling. The even- and odd-mode effective dielectric constants are much different when good coupling occurs, and simply averaging the two worked well in the cases discussed in 


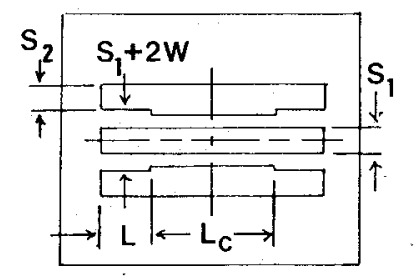

(a)

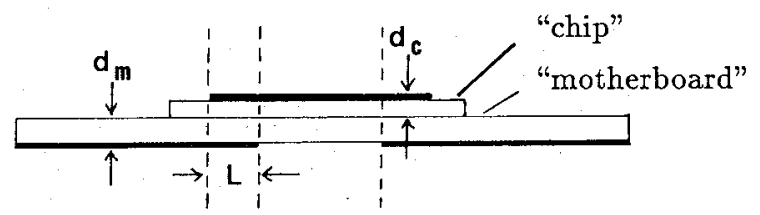

(b)

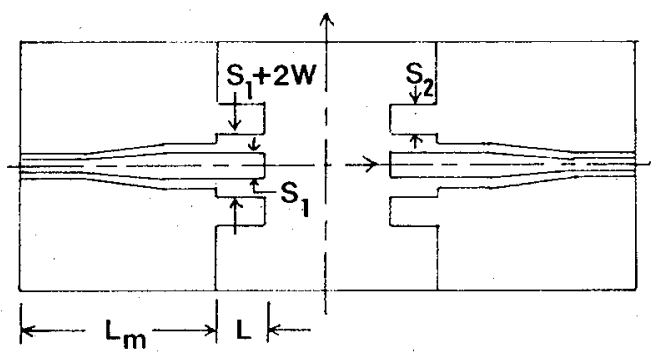

(c)

Fig. 5. Test configuration; (a) top view of chip, (b) cross section, and (c) bottom view of motherboard with dimensions of $S_{1}=2.4 \mathrm{~mm}$, $S_{2}=4.8 \mathrm{~mm}, W=3.9 \mathrm{~mm}, L=6.3 \mathrm{~mm}, L_{c}=13.0 \mathrm{~mm}, L_{m}=4.5 \mathrm{~cm}$, $d_{m}=1.27 \mathrm{~mm}$, and $\dot{d}_{c}=0.635 \mathrm{~mm}$.

this paper. Equations which more properly account for the different phase velocities can be found in the literature [7].

By making $d_{1}$ nonzero and setting $\epsilon_{r 1}=1.0$, the effect of an air gap of $2 d_{1}$ can be estimated. Fig. 4 shows that an air gap primarily affects the odd-mode effective permittivity. This is not surprising since the electric fields of the odd mode are more normal to the dielectric-air interface than the electric fields of the even mode (see Fig. 2(a) and (b)).

\section{MEASURED RESUlts}

These types of transitions have been designed, built, and measured at $5 \mathrm{GHz}$ and $10 \mathrm{GHz}$. Fig. 5 shows the dimensions of a set of $5-\mathrm{GHz}$ couplers built on Duroid substrate $\left(\epsilon_{r}=10.2\right)$.. There are two transitions, one from the "motherboard" (1.27 mm thick) to the "chip" (0.635 mm) and one back again to the motherboard. Measured return loss, shown in Fig. 6, is better than $20 \mathrm{~dB}$ over a 25 -percent bandwidth. This return loss is due to the two surfaceto-surface transitions and has been de-embedded from the coax-to-CPW transitions on either end of the motherboard using the time-domain option on an HP-8510 system. The insertion loss of the entire structure (including coax transitions) is about $0.6 \mathrm{~dB}$ and is well behaved in the passband.

A $10-\mathrm{GHz}$ single transition from one side of a substrate (Duroid $6010.2,1.27 \mathrm{~mm}$ ) to the other was constructed and measured to have better than $15 \mathrm{~dB}$ of return loss over a
SEF -20.0 IB $5.0 \mathrm{~dB} /$

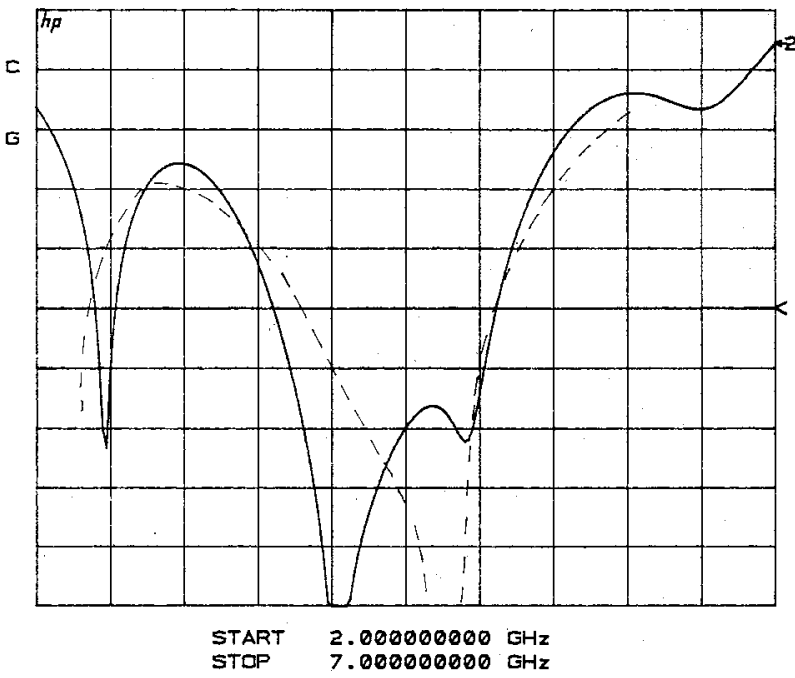

Fig. 6. Measured (__- ) and calculated (---) $\left|S_{11}\right|$ for test configura tion.

10-percent bandwidth. This includes the coax-to-CPW transitions at either end of the substrate.

In both cases, the center of the passband was measured to be about 12 percent lower than the design frequency. This is attributed to the open end discontinuities which make the coupled line sections look electrically longer than their physical length. The dashed line in Fig. 6 is the frequency response of the theoretical model of the measured structure. This model consists of two ideal transitions (described by (1)) separated by an ideal transmission line of appropriate electrical length. The modeled length of each coupler is in excess of the physical length by about $1.2 \mathrm{~mm}$ and was chosen to match the theoretical and measured center frequencies. Note that the measured and theoretical bandwidths are in fair agreement and that length extensions of $\Delta l=0.6 \mathrm{~mm}$ on each end of the ideal coupler are sufficient to compensate for fringing fields in the actual coupler. In an attempt to reduce this end effect, the chip substrate was trimmed so that the open ends terminated at the substrate edge. This produced a marked deterioration in match due to the fact that trimming reduced the end capacitance for the open end on the chip but not for the open end on the motherboard. Simple circuit modeling shows that an imbalance like this will produce the effect observed.

The sensitivity of the $5-\mathrm{GHz}$ model was investigated by displacing the chip enough to cause a significant change in the de-embedded return loss. A transverse displacement of roughly $1.3 \mathrm{~mm}(2 / 3$ of the combined substrate thickness) reduced the return loss from more than $20 \mathrm{~dB}$ to roughly $15 \mathrm{~dB}$. A longitudinal displacement of roughly $\pm 1.8 \mathrm{~mm}$ caused the same change in return loss. Generally, it was found that the transition was not overly sensitive to alignment.

As stated previously, the presence of a low-permittivity substrate below or above the coupled line region has little 
effect. This was confirmed by pressing a $1.5-\mathrm{mm}$-thick $\epsilon_{r}=2.5$ substrate on the underside of the structure in Fig. 5. Measured return loss changed only slightly and was still better than $17 \mathrm{~dB}$ over a 25-percent bandwidth. More discussion of this is presented below.

\section{Discussion}

One of the principal concerns in the use of this type of transition will be its size. The width of the structure is controlled by the substrate thickness. By reducing the separation (substrate thickness) between launching and receiving lines, one can reduce the size necessary for a given coupling. In Fig. 1(b) the feed network is on the same side of the motherboard as the chip, and the separation of the two coupled lines is just the thickness of the chip substrate. If, for example, the chip were $0.635-\mathrm{mm}$ thick alumina and the motherboard were $1.27-\mathrm{mm}$ quartz, the transverse dimensions for a $50-\Omega$ match would be reduced to 40 percent of those in Fig. 5. Of course the up-down symmetry is broken by placing the conductors in this manner, but if the motherboard permittivity is much lower than the chip permittivity, this effect is small. A rough estimate of the effect of motherboard presence can be seen by computing the odd and even impedances of Fig. 3 if $d_{3}$ is made to be half the chip thickness, $d_{2}$ is the motherboard thickness, $d_{1}$ is infinite, and the permittivities are arranged appropriately. For the alumina/quartz example already mentioned, this increased the dimensions from roughly 33 percent to 40 percent of Fig. 4 .

The longitudinal size is primarily determined by the operating frequency since the coupling region is one quarter of an effective wavelength long. In terms of coupling to MMIC's, this size requirement means that practical applications of this transition will be the millimeter-wave range.

At $35-\mathrm{GHz}$, a $50-\Omega$ transition from a $0.25-\mathrm{mm}$ quartz motherboard to a $0.1-\mathrm{mm}$ GaAs chip is computed to have the following dimensions (see Fig. 3). $S_{1}=50 \mu \mathrm{m}, S_{1}+2 \mathrm{~W}$ $=500 \mu \mathrm{m}, S_{2}=450 \mu \mathrm{m}$, and a length of $790 \mu \mathrm{m}$. These dimensions are much the same for a transition at $60 \mathrm{GHz}$ except that the length is about halved. These sizes indicate that transitions such as this would probably be too large to use with current MMIC's $(1 \mathrm{~mm} \times 2 \mathrm{~mm})$ but may have applications for the larger IC's which will be used in the future [1].

\section{Conclusions}

A transition has been developed for coupling coplanar waveguide on one surface to coplanar waveguide on another. Coupled line theory and a full-wave analysis were used to predict the behavior of this transition. Measured results show good agreement with theory insofar as match is concerned, but measured center frequency was lower than predicted due to end effects. A 25-percent bandwidth was measured.

This transition is likely to have applications in hybrid circuits at microwave frequencies or in large integrated circuits or layers of circuits at millimeter-wave frequencies.

\section{APPENDIX}

The fields in each layer of Fig. 3 can be obtained from the $z$-directed magnetic and electric potentials in each of the three layers. For electric cover plates,

$$
\begin{aligned}
\psi_{1}^{m} & =C_{1}^{m} \cos k_{1}\left(d_{1}+d_{2}+z\right) \\
\psi_{1}^{e} & =C_{1}^{e} \sin k_{1}\left(d_{1}+d_{2}+z\right) \\
\psi_{2}^{m} & =C_{2}^{m} \cos k_{2} z+D_{2}^{m} \sin k_{2} z \\
\psi_{2}^{e} & =C_{2}^{e} \cos k_{2} z+D_{2}^{e} \sin k_{2} z \\
\psi_{3}^{m} & =C_{3}^{m} \cos k_{3}\left(d_{3}-z\right) \\
\psi_{3}^{e} & =C_{3}^{e} \sin k_{3}\left(d_{3}-z\right)
\end{aligned}
$$

where $k_{t}=\left(\epsilon_{i} k_{0}^{2}-k_{x}^{2}-k_{y}^{2}\right)^{1 / 2}$, the subscripts $i=1,2,3$ refer to the different layers, and

$$
\begin{aligned}
C_{2}^{m}= & \frac{\mathrm{TM}_{2}\left(k_{3} / \epsilon_{3}\right) \sin k_{3} d_{3}}{\mathrm{TM}_{2}\left(k_{3} / \epsilon_{3}\right) \sin k_{3} d_{3}+\mathrm{TM}_{1}\left(k_{2} / \epsilon_{2}\right) \cos k_{3} d_{3}} \\
& \cdot \frac{j\left(k_{x} \tilde{J}_{x}+k_{y} \tilde{J}_{y}\right)}{k_{x}^{2}+k_{y}^{2}} \\
C_{2}^{e}= & \frac{\mathrm{TE}_{2} \sin k_{3} d_{3}}{\mathrm{TE}_{2} k_{3} \cos k_{3} d_{3}+\mathrm{TE}_{1} k_{2} \sin k_{3} d_{3}} \\
& \cdot \frac{\omega \mu_{0}\left(k_{y} \tilde{J}_{x}-k_{x} \tilde{J}_{y}\right)}{k_{x}^{2}+k_{y}^{2}} \\
D_{2}^{m}= & -C_{2}^{m} \mathrm{TM}_{1} / \mathrm{TM}_{2} \\
C_{1}^{m}= & \left(C_{2}^{m} \cos k_{2} d_{2}-D_{2}^{m} \sin k_{2} d_{2}\right) / \cos k_{1} d_{1} \\
C_{1}^{e}= & \left(C_{2}^{e} \cos k_{2} d_{2}-D_{2}^{e} \sin k_{2} d_{2}\right) / \sin k_{1} d_{1} \\
C_{3}^{e}= & C_{2}^{e} / \sin k_{3} d_{3} \\
C_{3}^{m}= & \left(k_{2} / \epsilon_{2}\right)\left(\epsilon_{3} / k_{3}\right) D_{2}^{m} / \sin k_{3} d_{3} \\
\mathrm{TM}_{1}= & \left(k_{2} / \epsilon_{2}\right) \cos k_{1} d_{1} \sin k_{2} d_{2} \\
& +\left(k_{1} / \epsilon_{1}\right) \sin k_{1} d_{1} \cos k_{2} d_{2} \\
\mathrm{TM}_{2}= & \left(k_{2} / \epsilon_{2}\right) \cos k_{1} d_{1} \cos k_{2} d_{2} \\
& -\left(k_{1} / \epsilon_{1}\right) \sin k_{1} d_{1} \sin k_{2} d_{2} \\
\mathrm{TE}_{1}= & k_{1} \cos k_{1} d_{1} \cos k_{2} d_{2}-k_{2} \sin k_{1} d_{1} \sin k_{2} d_{2} \\
\mathrm{TE}_{2}= & k_{1} \cos k_{1} d_{1} \sin k_{2} d_{2}+k_{2} \sin k_{1} d_{1} \cos k_{2} d_{2} . \quad(\mathrm{A} 2) \\
&
\end{aligned}
$$

For magnetic cover plates the potentials are

$$
\begin{aligned}
\psi_{1}^{\prime m} & =C_{1}^{\prime m} \sin k_{1}\left(d_{1}+d_{2}+z\right) \\
\psi_{1}^{\prime e} & =C_{1}^{\prime e} \cos k_{1}\left(d_{1}+d_{2}+z\right) \\
\psi_{2}^{\prime m} & =C_{2}^{\prime m} \cos k_{2} z+D_{2}^{\prime m} \sin k_{2} z \\
\psi_{2}^{\prime e} & =C_{2}^{\prime e} \cos k_{2} z+D_{2}^{\prime e} \sin k_{2} z \\
\psi_{3}^{\prime m} & =C_{3}^{\prime m} \sin k_{3}\left(d_{3}-z\right) \\
\psi_{3}^{\prime e} & =C_{3}^{\prime e} \cos k_{3}\left(d_{3}-z\right)
\end{aligned}
$$


where the prime denotes magnetic cover plates and

$$
\begin{aligned}
C_{2}^{\prime m}= & \frac{\mathrm{TM}_{1}^{\prime}\left(k_{3} / \epsilon_{3}\right) \cos k_{3} d_{3}}{\mathrm{TM}_{1}^{\prime}\left(k_{3} / \epsilon_{3}\right) \cos k_{3} d_{3}-\mathrm{TM}_{2}^{\prime}\left(k_{2} / \epsilon_{2}\right) \sin k_{e} d_{3}} \\
& \cdot \frac{\left(k_{x} \tilde{J}_{x}+k_{y} \tilde{J}_{y}\right)}{\left(k_{x}^{2}+k_{y}^{2}\right)} \\
C_{2}^{\prime e}= & \frac{\mathrm{TE}_{1}^{\prime} \cos k_{3} d_{3}}{\mathrm{TE}_{2}^{\prime} k_{2} \cos k_{3} d_{3}-\mathrm{TE}_{1}^{\prime} k_{3} \sin k_{3} d_{3}} \\
& \cdot \frac{\omega \mu_{0}\left(k_{y} \tilde{J}_{x}-k_{x} \tilde{J}_{y}\right)}{\left(k_{x}^{2}+k_{y}^{2}\right)} \\
D_{2}^{\prime m}= & -C_{1}^{\prime m} \mathrm{TM}_{2}^{\prime} / \mathrm{TM}_{1}^{\prime} \\
C_{1}^{\prime m}= & \left(C_{2}^{\prime m} \cos k_{2} d_{2}-D_{2}^{\prime m} \sin k_{2} d_{2}\right) / \sin k_{1} d_{1} \\
C_{1}^{\prime e}= & \left(C_{2}^{\prime e} \cos k_{2} d_{2}-D_{2}^{\prime e} \sin k_{2} d_{2}\right) / \cos k_{1} d_{1} \\
C_{3}^{\prime e}= & C_{2}^{\prime e} / \cos k_{3} d_{3} \\
C_{3}^{\prime m}= & -\left(\epsilon_{3} / k_{3}\right)\left(k_{2} / \epsilon_{2}\right) D_{2}^{\prime m} / \cos k_{3} d_{3} \\
\mathrm{TM}_{1}^{\prime}= & \left(k_{2} / \epsilon_{2}\right) \cos k_{2} d_{2} \sin k_{1} d_{1} \\
& +\left(k_{1} / \epsilon_{1}\right) \cos k_{1} d_{1} \sin k_{2} d_{2} \\
\mathrm{TM}_{2}^{\prime}= & \left(k_{2} / \epsilon_{2}\right) \sin k_{1} d_{1} \sin k_{2} d_{2} \\
& -\left(k_{1} / \epsilon_{1}\right) \cos k_{1} d_{1} \cos k_{2} d_{2} \\
\mathrm{TE}_{1}^{\prime}= & k_{1} \sin k_{1} d_{1} \sin k_{2} d_{2}-k_{2} \cos k_{2} d_{2} \cos k_{1} d_{1} \\
\mathrm{TE}_{2}^{\prime}= & k_{1} \sin k_{1} d_{1} \cos k_{2} d_{2}+k_{2} \sin k_{2} d_{2} \cos k_{1} d_{1} . \quad(\mathrm{A} 4)
\end{aligned}
$$

The Fourier series field amplitudes are then obtained from

$$
\begin{aligned}
& \tilde{\overrightarrow{E_{i}}}=-\tilde{\vec{\nabla}} \times \psi_{i}^{e} \hat{z}+\frac{1}{j \omega \epsilon_{0} \epsilon_{i}} \tilde{\vec{\nabla}} \times \tilde{\vec{\nabla}} \times \psi_{i}^{m} \hat{z} \\
& \tilde{\overrightarrow{H_{i}}}=\tilde{\vec{\nabla}} \times \psi_{i}^{m} \hat{z}+\frac{1}{j \omega \mu_{0}} \tilde{\vec{\nabla}} \times \tilde{\vec{\nabla}} \times \psi_{i}^{e} \hat{z}
\end{aligned}
$$

where

$$
\tilde{\vec{\nabla}}=j k_{x} \hat{x}+j k_{y} \hat{y}+\hat{z} \frac{\partial}{\partial z} .
$$

Equations (2) are easily obtained from the preceding expressions by replacing $k_{x}$ by $\beta$ and $k_{y}$ by $n \pi / a$.

\section{REFERENCES}

[1] J. A. Kinzel, "GaAs technology for millimeter-wave phased arrays," IEEE Antennas Propagat. Newsletter, vol. 29, pp. 12-14, Feb. 1987.

[2] R. W. Jackson, "Considerations in the use of coplanar waveguide for millimeter-wave integrated circuits," IEEE Trans. Microwave Theory Tech., vol. MTT-34, pp. 1450-1456, Dec. 1986.

[3] M. Riaziat, I. Zubeck, S. Bandy, and G. Zdasiuk, "Coplanar waveguide used in 2-18 $\mathrm{GHz}$ distributed amplifier," in IEEE MTT-S Microwave Symp. Dig., 1986, pp. 337-338.
[4] G. Matthaei, L. Young, and E. M. T. Jones, Microwave Filters, Impedance-Matching Networks, and Coupling Structures. Dedham, MA: Artech House, 1980, p. 221.

[5] R. Jansen, "Unified user oriented computation of shie]ded, covered and open planar microwave and millimeter wave transmission line characteristics," Microwave, Opt., Acoust., vol. 3, pp. 14-22, Jan. 1979.

[6] T. Itoh, "Spectral-domain immittance approach for dispersion characteristics of generalized printed transmission lines," IEEE Trans. Microwave Theory Tech., vol. MTT-28, pp. 733-736, July 1980.

[7] G. I. Zysman and A. K. Johnson, "Coupled transmission line networks in an inhomogeneous dielectric medium,"IEEE Trans. Microwave Theory Tech., vol. MTT-28, pp. 733-736, July 1980.

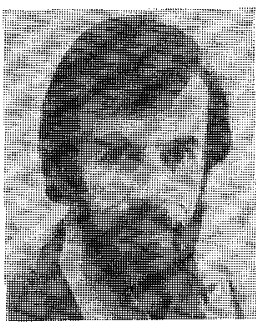

Robert W. Jackson (M'82) was born in Boston, MA, on October 18, 1952. he received the B.S. degree in 1975 and the Ph.D. degree in 1981, both in electrical engineering, from Northeastern University in Boston. His thesis wás on nonlinear plasma interactions in the earth's bow shock.

From 1981 to 1982 he was an Assistant Professor at Northeastern University. Since 1982, he has been on the faculty of the Department of Electrical and Computer Engineering at the University of Massachusetts, Amberst, where he is a member of the Microwave and Electronics Laboratory. His research interests include numerical electromagnetics applied to millimeter-wave integrated circuits and active microwave and millimeter-wave circuit design.

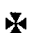

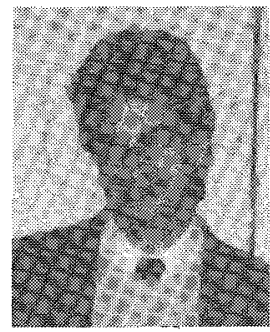

David W. Matolak (S'85-M'86) was born in Johnstown, PA, on August 29, 1961. He received the B.S.E.E. degree from the Pennsylvania State University in 1983 and the M.S.E.C.E. degree from the University of Massachusetts in 1987.

From September 1983 to January 1985, he was employed in the Telecommunications Engineering and Standards Division of the Fural Electrification Administration, Washington, DC, where he analyzed low-capacity microwave radio systems and digital voice-processing techniques. From January 1985 to October 1986, he was a graduate student and research assistant in the Microwave and Electronics Laboratory of the University of Massachusetts. In October 1986, he joined AT\&T Bell Laboratories, North Andover, MA, where he is working on the analysis of analog and digital microwave radio systems.

Mr. Matolak is a member of Eta Kappa Nu. 